\title{
COMUNIDADE ARBÓREA DE UMA FLORESTA ESTACIONAL DECÍDUA SOBRE AFLORAMENTO CALCÁRIO NA BACIA DO RIO PARANÁ ${ }^{1}$
}

\author{
Luciana Álvares da Silva ${ }^{2}$ e Aldicir Scariot ${ }^{3}$
}

\begin{abstract}
RESUMO - A bacia do rio Paraná (GO e TO), com 5.940.382 ha, tem alta diversidade de fitofisionomias, com sua flora pouco conhecida. As atividades de extração de madeira e implantação de pastagem contribuíram decisivamente para a remoção da vegetação. Este estudo foi conduzido em uma floresta estacional decídua sobre afloramento calcário (aproximadamente 1341'16"S e 4644'20"W e 462 m de altitude) - fazenda Canadá (São DomingosGO). Foram demarcadas cinco linhas paralelas, a intervalos de $100 \mathrm{~m}$, onde foram distribuídas aleatoriamente 25 parcelas de $20 \times 20 \mathrm{~m}$ (total de 1 ha). Em cada parcela foram amostradas todas as árvores com diâmetro do caule a 1,3 m de altura do solo (DAP) $\geq 5 \mathrm{~cm}$, nos quais foi medido o DAP, estimada a altura máxima e identificada a espécie. Foram amostrados 924 indivíduos de 48 espécies, 38 gêneros e 24 famílias e obtidos o índice de Shannon-Wienner de 2,99 nats/ind. e a equabilidade de 0,77. As espécies que apresentaram maior valor de importância (VI) foram Myracrodruon urundeuva (36,09), Pseudobombax tomentosum (34,75), Dilodendron bipinnatum (26,61), Combretum duarteanum (22,19), Jacaranda brasiliana (21,57), Commiphora leptophloeos $(19,18)$, Astronium fraxinifolium $(13,84)$, Tabebuia impetiginosa $(13,79)$, Pseudobombax longiflorum $(11,64) \mathrm{e}$ Machaerium scleroxylon (10,00), que juntas somaram 69,9\% do VI total. A diversidade foi próxima à encontrada em outros trabalhos em floresta estacional decídua sobre solo e afloramento na região.
\end{abstract}

Palavras-chave: Fitossociologia e floresta estacional decidual.

\section{ARBOREAL COMMUNITY OF A SEASONAL DECIDUOUS FOREST ON LIMESTONE OUTCROP IN SÃO DOMINGOS - GOIÁS, PARANA RIVER BASIN, BRAZIL}

\begin{abstract}
The Parana River basin - an area of 5,940,382 ha located in Brazil- has a highly diverse phytophysiognomy and scarce flora information. Wood extraction and pasture activities have decisively contributed to the removal of vegetation in this area. This study was carried out in a seasonal deciduous forest on limestone

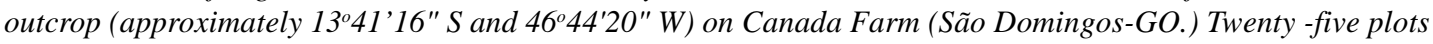
of $20 \mathrm{~m} \times 20 \mathrm{~m}(1 \mathrm{ha})$ were randomly allocated on parallel lines $100 \mathrm{~m}$ apart. Trees with stem diameter at $1.3 \mathrm{~m}$ breast height $(D B H) \geq 5 \mathrm{~cm}$ were sampled and their $D B H$, maximum height and species determined. In the phytosociological study, 924 individuals comprising 48 species, 38 genera and 24 families were sampled. The species with the highest importance value (IV) were Myracrodruon urundeuva (36.09\%), Pseudobombax tomentosum (34.75\%), Dilodendron bipinnatum (26.61\%), Combretum duarteanum (22.19\%), Jacaranda brasiliana (21.57\%), Commiphora leptophloeos (19.18\%), Astronium fraxinifolium (13.84\%), Tabebuia impetiginosa (13.79\%), Pseudobombax longiflorum (11.64\%) and Machaerium scleroxylon (10.00\%), comprising $69.9 \%$ of the total IV. Diversity was close to that found in other seasonal deciduous forest studies in the region.
\end{abstract}

Key words: $\quad$ Phytosociology and seasonal deciduous forest.

1 Recebido para publicação em 25.2.2002 e aceito para publicação em 17.2.2004.

2 Bolsista Recém-Doutor, CNPq/Embrapa Recursos Genéticos e Biotecnologia, PqEB - Parque Estação Biológica W5 Norte Final, Caixa Postal 02372, 70770-900 Brasília-DF, <luciana.alvares@zipmail.com.br>; ${ }^{3}$ Pesquisador da Embrapa Recursos Genéticos e Biotecnologia, <scariot@ cenargen.embrapa.br> 


\section{INTRODUÇÃO}

A fragmentação florestal é um dos principais problemas que afetam as florestas tropicais atualmente (Whitmore, 1997). Os efeitos da fragmentação são relacionados não somente à redução da área, mas também ao aumento no isolamento entre fragmentos remanescentes (Skole \& Tucker, 1993), causando alterações físicas e bióticas em uma floresta previamente intacta (Lovejoy et al., 1983; Bierregaard et al., 1992).

As florestas estacionais decíduas no domínio do cerrado encontram-se distribuídas nos Estados de Minas Gerais, Goiás, Mato Grosso, Bahia e no Distrito Federal, em forma de manchas (Pedralli, 1997). Apesar de estarem localizadas em uma paisagem bastante antropizada, apresentam alta riqueza de espécies de importância econômica e ainda são exploradas para retirada de madeira e para ampliação de áreas de pastagens e agricultura (Scariot \& Sevilha, 2000).

Nos últimos dois séculos, as florestas estacionais decíduas foram reduzidas a pequenos fragmentos, geralmente perturbados, onde grandes áreas foram desmatadas para implantação de agricultura e pastagens. (Gerhardt, 1994; Werneck et al., 2000).

A bacia do rio Paraná está localizada parte em Goiás e parte em Tocantins, apresentando alta diversidade de fitofisionomias em meio a uma matriz antropizada, com fragmentos de vegetação de diversos tamanhos e níveis de perturbação causados pelo homem, destacando-se os fragmentos naturais representados pelos afloramentos calcários. As florestas estacionais decíduas sobre afloramentos são muito comuns na região e ainda encontramse bem preservadas, devido à dificuldade de extração de madeira, enquanto os fragmentos de floresta estacional decídua sobre solos, geralmente perturbados, remanescem nas áreas planas, em meio à pastagem (Scariot \& Sevilha, 2000).

A floresta estacional decídua sobre solos litólicos cascalhentos de origem calcária é considerada de relevante importância, uma vez que apresenta flora e fisionomia própria, bem distinta de outras formações sobre outros tipos de solos (Pedersoli \& Martins, 1972; Ivanauskas \& Rodrigues, 2000). São necessários estudos com vistas ao conhecimento de sua biodiversidade e ações de preservação, uma vez que apresentam particularidades florísticas pronunciadas (Ivanauskas \& Rodrigues, 2000).

R. Árvore, Viçosa-MG, v.28, n.1, p.61-67, 2004
Os objetivos deste trabalho foram o levantamento quantitativo do componente arbóreo para caracterizar uma área de floresta estacional decídua sobre afloramento calcário na região da bacia do rio Paraná (Goiás e Tocantins) e fornecer subsídios para estratégias de conservação.

\section{MATERIAL E MÉTODOS}

A região da bacia do rio Paraná é circundada por relevos de planaltos e chapadas, constituindo uma região deprimida, alongada no sentido norte-sul, com altitudes entre 400 e 600 m (IBGE, 1995).

Este estudo foi feito em área de floresta estacional decídua sobre afloramento calcário na fazenda Canadá, município de São Domingos, Goiás (bacia do rio Paraná). $\mathrm{O}$ afloramento está aproximadamente a 13\%4'16" S e 46 $44^{\prime} 20^{\prime \prime} \mathrm{W}$, a uma altitude média de $462 \mathrm{~m}$, e tem cerca de $300 \times 600 \mathrm{~m}$.

O clima, segundo a classificação de Köppen, é do tipo $\mathrm{Cw}$, com médias anuais de temperatura de $24^{\circ} \mathrm{C}$, pluviosidade de $1.500 \mathrm{~mm}$ e umidade relativa de $60 \%$, sob duas estações climáticas bem definidas, sendo uma chuvosa, seguida por longo período seco.

A vegetação é do tipo floresta estacional decídua, apresentando mais de 50\% das árvores do dossel ou emergentes sem folhas durante o período seco (Veloso et al., 1991).

Para amostragem da vegetação foram demarcadas cinco linhas paralelas, ao longo da inclinação do terreno do afloramento, a uma distância de 100 m entre as linhas. Nessas linhas foram distribuídas, aleatoriamente, 25 parcelas de $20 \times 20 \mathrm{~m}\left(400 \mathrm{~m}^{2}\right)$, totalizando 1 ha amostrado (Figura 1).

Em cada parcela foi colocada uma estaca com a identificação da linha e o número da parcela. Devido às dificuldades de se fixar a estaca na rocha, as áreas das parcelas foram demarcadas com tinta vermelha sobre a rocha, para facilitar visitas futuras. Nas parcelas, todos os indivíduos arbóreo-arbustivos com diâmetro do caule a 1,3 m de altura do solo (DAP) maior ou igual a $5 \mathrm{~cm}$ foram etiquetados com plaquetas de alumínio, identificados e tiveram o DAP medido e a altura máxima estimada. Os indivíduos que apresentaram troncos múltiplos foram incluídos na amostragem quando os perfilhos atingiam o diâmetro mínimo estabelecido. Para os indivíduos não-identificados, foram coletados materiais 
férteis, quando possível. O material botânico coletado foi identificado com a ajuda de especialistas e por meio de comparação no herbário do Cenargen e na literatura. As exsicatas foram incorporadas ao herbário do Cenargen (CEN).

O índice de diversidade de Shannon-Wienner ( $\left.\mathrm{H}^{\prime}\right)$ e a equabilidade de Pielou (J') foram calculados conforme Magurran (1987), e os parâmetros da estrutura fitossociológica da comunidade foram calculados de acordo com Mueller-Dombois \& Ellenberg (1974).

Os indivíduos amostrados foram distribuídos em classes de altura, cuja distribuição de freqüência foi elaborada na forma de histogramas. A determinação do número mínimo de classes e a amplitude foram estabelecidas de acordo com Spiegel (1976).

\section{RESULTADOS E DISCUSSÃO}

No levantamento foram encontrados 924 indivíduos arbóreos, distribuídos em 24 famílias, 38 gêneros e
48 espécies. As espécies amostradas foram colocadas em ordem alfabética de família e espécie (Quadro 1).

Nas áreas de afloramento, o substrato rochoso e o estresse hídrico na época seca são os principais fatores que selecionam a ocorrência de espécies, resultando na diminuição da riqueza.

Algumas espécies encontradas na amostragem também ocorreram em outros levantamentos em áreas de afloramentos calcários nos Estados de São Paulo e Minas Gerais e no Distrito Federal (Ivanauskas \& Rodrigues, 2000; Mecenas et al., 1996; Pedrali, 1997). Dentre elas podem ser citadas: Astronium fraxinifolium, Aspidosperma subincanum, Commiphora leptophloeos, Dilodendron bipinatum, Enterolobium contortisiliquum, Guazuma ulmifolia, Luehea divaricata, Machaerium scleroxylon, Machaerium stipitatum, Machaerium villosum, Maclura tinctoria, Myracrodruon urundeuva, Platypodium elegans, Pseudobombax longiflorum, Rhamnidium elaeocarpum, Schinopsis brasiliensis, Simira sampaioana, Tabebuia impetiginosa e Tabebuia roseo-alba.

Quadro 1 - Relação das espécies amostradas no levantamento da estrutura da comunidade arbórea, em uma floresta estacional decídua em afloramento calcário, no município de São Domingos - GO, bacia do rio Paraná. Famílias e espécies em ordem alfabética

Table 1 - List of the tree species sampled in a deciduous forest on a limestone outcrop in São Domingos - Goiás, Paraná River Basin, Brazil. Family and species are listed in alphabetical order

\begin{tabular}{|c|c|c|}
\hline Família & Nome Científico & Nome Popular \\
\hline \multirow{3}{*}{ Anacardiaceae } & Astronium fraxinifolium Schott & Gonçalo \\
\hline & Myracrodruon urundeuva Allemão & Aroeira \\
\hline & Schinopsis brasiliensis Engl. & Braúna \\
\hline \multirow{4}{*}{ Apocynaceae } & Aspidosperma cuspa (Kunth) S.F. Blake & Pereiro \\
\hline & Aspidosperma pyrifolium Mart. & Peroba \\
\hline & Aspidosperma sp. & Pereiro-rabo-de-tatu \\
\hline & Aspidosperma subincanum Mart. & "'- \\
\hline \multirow{4}{*}{ Bignoniaceae } & Jacaranda brasiliana (Lam.) Pers. & Caroba \\
\hline & Tabebuia aurea (Silva Manso) Benth. \& Hook. & Pau-d'árco \\
\hline & Tabebuia impetiginosa (Mart.) Standl. & Ipế \\
\hline & Tabebuia roseo-alba (Ridl.) Sandwith & Taipoca \\
\hline \multirow{4}{*}{ Bombacaceae } & Cavanillesia arborea $\mathrm{K}$. Schum. & Barriguda \\
\hline & Chorisia pubiflora (A. St.-Hil.) E.Y. Dawson. & Barrigudinha \\
\hline & Pseudobombax longiflorum (Mart. \& Zucc.) A. Robyns & Canudeiro \\
\hline & Pseudobombax tomentosum (Mart. \& Zucc.) A. Robyns & Imbiruçu \\
\hline Boraginaceae & Cordia glabrata (Mart.) DC. & Cordia \\
\hline \multirow{2}{*}{ Burseraceae } & Commiphora leptophloeos (Mart.) J.B.Gillet & Cambão \\
\hline & Commiphora sp. & Falsa-cerejeira \\
\hline Cecropiaceae & Cecropia saxatilis Snethl. & Embaúba \\
\hline Celastraceae & Maytenus floribunda Reissek & - \\
\hline
\end{tabular}


Quadro 1, cont. Table 1, cont.

\begin{tabular}{|c|c|c|}
\hline Família & Nome Científico & Nome Popular \\
\hline \multirow{2}{*}{ Combretaceae } & Combretum duarteanum Cambess. & Vaqueta \\
\hline & Terminalia argentea Mart. \& Zucc. & - \\
\hline \multirow{2}{*}{ Euphorbiaceae } & Piranhea securinega A.Radcl.-Sm. \& Ratter & - \\
\hline & Jatropha sp. & - \\
\hline Leguminosae Caesalpinioideae & Bauínia acuruana Moric. & Bauínia \\
\hline \multirow{3}{*}{ Leguminosae Mimosoideae } & Acacia glomerosa Benth. & Piriquiteira \\
\hline & Anadenanthera peregrina (L.) Speg. & Angico \\
\hline & Enterolobium contortisiliquum (Vell.) Morong. & Tamboril \\
\hline \multirow{6}{*}{ Leguminosae Papilionoideae } & Luetzelburgia $\mathrm{sp}$. & Cabeludinha \\
\hline & Machaerium angustifolium Vogel & Jacarandá-de-espinho \\
\hline & Machaerium scleroxylon Tul. & Pau-ferro \\
\hline & Machaerium stipitatum (DC.) Vogel & - \\
\hline & Machaerium villosum Vogel & Jacarandá \\
\hline & Platypodium elegans Vogel & - \\
\hline Malpighiaceae & Não-identificada & Canela-de-velho \\
\hline \multirow{2}{*}{ Moraceae } & Ficus insipida Willd. & Ficus-amarelinho \\
\hline & Maclura tinctoria (L.) D. Don ex Steud. & Amoreira \\
\hline Olacaceae & Ximenia americana $\mathrm{L}$. & Ameixa \\
\hline Polygonaceae & Triplaris gardneriana Wedd. & Pau-jaú \\
\hline Rhamnaceae & Rhaminidium elaeocarpum Reissek & - \\
\hline \multirow{2}{*}{ Rubiaceae } & Coutarea sp. & - \\
\hline & Simira sampaioana (Standl.) Steyerm. & - \\
\hline Sapindaceae & Dilodendron bipinnatum Radlk. & Mamoninha \\
\hline Simaroubaceae & Simarouba versicolor A. St.-Hil. & - \\
\hline \multirow{2}{*}{ Sterculiaceae } & Guazuma ulmifolia Lam. & Mutamba \\
\hline & Sterculia striata A. St.-Hil. \& Naudin & Chichá \\
\hline Tiliaceae & Luehea divaricata Mart. & Açoita-cavalo \\
\hline Vochysiaceae & Callisthene fasciculata (Spreng.) Mart. & Pau-jacaré \\
\hline
\end{tabular}

As famílias com maiores números de espécies foram Fabaceae (6 espécies), Apocynaceae (4), Bignoniaceae (4) e Bombacaceae (4), correspondendo a $37,5 \%$ das espécies encontradas (Quadro 1). Em número de indivíduos, as famílias mais abundantes foram Anacardiaceae (181 indivíduos), Bignoniaceae (124), Combretaceae (110), Sapindaceae (105) e Bombacaceae (102), perfazendo $67,3 \%$ do total de indivíduos amostrados.

As espécies com maiores valores de importância (VI) foram Myracrodruon urundeuva $(36,09)$, Pseudobombax tomentosum $(34,75)$, Dilodendron bipinnatum $(26,61)$, Combretum duarteanum $(22,19)$, Jacaranda brasiliana (21,57), Commiphora leptophloeos $(19,18)$, Astronium fraxinifolium $(13,84)$, Tabebuia impetiginosa $(13,79)$, Pseudobombax longiflorum $(11,64)$ e Machaerium scleroxylon $(10,00)$, que juntas somaram 69,9\% do VI total (Quadro 2). Os maiores valores de VI foram devidos principalmente aos altos valores de densidade e dominância relativa das espécies. As espécies M. urundeuva, P. tomentosum, C. duarteanum, J. brasiliana, C. leptophloeos e T. impetiginosa também apresentaram maior VI em área de afloramento, encontrado por Silva \& Scariot (2003).

Para o valor de cobertura (VC), a posição alterou somente para as espécies $P$. longiflorum e A. fraxinifolium. Apesar de P. longiflorum apresentar baixo número de indivíduos, é uma espécie de maior porte que A. fraxinifolium, apresentando maior dominância.

O índice de diversidade de Shannon-Wienner ( $\left.\mathrm{H}^{\prime}\right)$ foi 2,99 nats/indivíduos e a equabilidade (J'), 0,77. Os valores são próximos aos encontrados em áreas de floresta estacional decídua sobre solo e sobre afloramentos de calcário, na mesma região estudada (Scariot \& Sevilha, 2000; Silva et al., 2001; Silva \& Scariot, 2001, 2003) e em área de afloramento na região de Piracicaba-SP (Ivanaukas \& Rodrigues, 2000) (Quadro 3). 
Quadro 2 - Parâmetros fitossociológicos das espécies amostradas no levantamento da estrutura da comunidade arbórea, em ordem de valor de importância (VI), em que $\mathrm{n}_{\mathrm{i}}=$ número de indivíduos da espécie; $\mathrm{N}_{\mathrm{i}}=$ número de parcelas onde a espécie está presente; $F A=$ freqüência absoluta; $F R=$ freqüência relativa; $D A=$ densidade absoluta; $D R=$ densidade relativa; $\mathrm{DoA}=$ dominância absoluta; $\mathrm{DoR}=$ dominância relativa; e VC = valor de cobertura

Table 2 - Phytosociological parameters of the species sampled in an arboreal community structure survey, following an importance value sequence $(V I)$ where $n_{i}=$ number of individuals of the species; $N_{i}=$ number of plots where the species occur; FA = absolute frequency; FR = relative frequency; DA = absolute density; DR = relative density; DoA $=$ absolute dominance; $\mathrm{DoR}=$ relative dominance; $\mathrm{VC}=$ cover value

\begin{tabular}{|c|c|c|c|c|c|c|c|c|c|c|}
\hline Espécie & $\mathrm{n}_{\mathrm{i}}$ & $\mathrm{N}_{\mathrm{i}}$ & FA & FR & DA & DR & DoA & DoR & VI & $\mathrm{VC}$ \\
\hline Myracrodruon urundeuva & 129 & 25 & 100,00 & 7,91 & 129,00 & 13,96 & 1,41 & 14,22 & 36,09 & 28,18 \\
\hline Pseudobombax tomentosum & 71 & 21 & 84,00 & 6,65 & 71,00 & 7,68 & 2,03 & 20,42 & 34,75 & 28,11 \\
\hline Dilodendron bipinnatum & 105 & 24 & 96,00 & 7,59 & 105,00 & 11,36 & 0,76 & $\begin{array}{l}7,65 \\
-3, m \rightarrow x\end{array}$ & 26,61 & 19,01 \\
\hline Combretum duarteanum & 109 & 19 & 76,00 & 6,01 & 109,00 & 11,80 & 0,43 & 4,38 & 22,19 & 16,18 \\
\hline Jacaranda brasiliana & 72 & 18 & 72,00 & 5,70 & 72,00 & 7,79 & 0,80 & 8,08 & 21,57 & 15,87 \\
\hline Commiphora leptophloeos & 68 & 20 & 80,00 & 6,33 & 68,00 & 7,36 & 0,54 & 5,49 & 19,18 & 12,85 \\
\hline Astronium fraxinifolium & 44 & 17 & 68,00 & 5,38 & 44,00 & 4,76 & 0,37 & $\begin{array}{l}3,70 \\
3,0\end{array}$ & 13,84 & 8,46 \\
\hline Tabebuia impetiginosa & 38 & 14 & 56,00 & 4,43 & 38,00 & 4,11 & 0,52 & 5,25 & 13,79 & 9,36 \\
\hline Pseudobombax longiflorum & 11 & 6 & 24,00 & 1,90 & 11,00 & 1,19 & 0,85 & $\begin{array}{l}8,55 \\
8,3\end{array}$ & 11,64 & 9,74 \\
\hline Machaerium scleroxylon & 31 & 12 & 48,00 & 3,80 & 31,00 & 3,35 & 0,28 & 2,84 & 10,00 & 6,20 \\
\hline Cordia glabrata & 24 & 13 & 52,00 & 4,11 & 24,00 & 2,60 & 0,23 & 2,28 & 8,99 & 4,88 \\
\hline Anadenanthera peregrina & 36 & 8 & 32,00 & 2,53 & 36,00 & 3,90 & 0,24 & 2,46 & 8,89 & 6,36 \\
\hline Chorisia pubiflora & 19 & 10 & 40,00 & 3,16 & 19,00 & 2,06 & 0,29 & 2,92 & 8,14 & 4,98 \\
\hline Luehea divaricata & 18 & 11 & 44,00 & 3,48 & 18,00 & 1,95 & 0,07 & 0,71 & 6,14 & 2,66 \\
\hline Simarouba versicolor & 14 & 7 & 28,00 & 2,22 & 14,00 & 1,52 & 0,16 & 1,64 & 5,37 & 3,15 \\
\hline Sterculia striata & 14 & 7 & 28,00 & 2,22 & 14,00 & 1,52 & 0,12 & 1,21 & 4,94 & 2,73 \\
\hline Luetzelburgia sp. & 13 & 5 & 20,00 & 1,58 & 13,00 & 1,41 & 0,15 & 1,54 & 4,53 & 2,94 \\
\hline Aspidosperma subincanum & 11 & 7 & 28,00 & 2,22 & 11,00 & 1,19 & 0,07 & 0,67 & 4,08 & 1,87 \\
\hline Aspidosperma cuspa & 9 & 7 & 28,00 & 2,22 & 9,00 & 0,97 & 0,03 & 0,33 & 3,51 & 1,30 \\
\hline Simira sampaioana & 6 & 8 & 32,00 & 2,53 & 6,00 & 0,65 & 0,03 & 0,31 & 3,49 & 0,96 \\
\hline Schinopsis brasiliensis & 8 & 5 & 20,00 & 1,58 & 8,00 & 0,87 & 0,05 & 0,48 & 2,93 & 1,34 \\
\hline Tabebuia aurea & 9 & 4 & 16,00 & 1,27 & 9,00 & 0,97 & 0,04 & 0,39 & 2,63 & 1,37 \\
\hline Tabebuia roseo-alba & 5 & 3 & 12,00 & 0,95 & 5,00 & 0,54 & 0,03 & 0,29 & 1,78 & 0,83 \\
\hline Ficus insipida & 4 & 3 & 12,00 & 0,95 & 4,00 & 0,43 & 0,04 & 0,38 & 1,77 & 0,82 \\
\hline Cecropia saxatilis & 4 & 3 & 12,00 & 0,95 & 4,00 & 0,43 & 0,04 & 0,37 & 1,75 & 0,80 \\
\hline Jatropha sp. & 6 & 2 & 8,00 & 0,63 & 6,00 & 0,65 & 0,04 & 0,43 & 1,71 & 1,07 \\
\hline Piranhea securinega & 5 & 3 & 12,00 & 0,95 & 5,00 & 0,54 & 0,02 & 0,20 & 1, & 0,74 \\
\hline Guazuma ulmifolia & 3 & 3 & 12,00 & 0,95 & 3,00 & 0,32 & 0,04 & 0,40 & $\begin{array}{l}1,67 \\
1,67\end{array}$ & 0,72 \\
\hline Triplaris gardneriana & 4 & 3 & 12,00 & 0,95 & 4,00 & 0,43 & 0,02 & 0,24 & 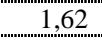 & 0,67 \\
\hline Machaerium villosum & 4 & 3 & 12,00 & 0,95 & 4,00 & 0,43 & 0,01 & 0,13 & $\begin{array}{l}1,52 \\
1,5, \ldots\end{array}$ & 0,57 \\
\hline Malpighiaceae & 4 & 3 & 12,00 & 0,95 & 4,00 & 0,43 & 0,01 & 0,10 & $\begin{array}{l}1,48 \\
1,4\end{array}$ & 0,53 \\
\hline Aspidosperma sp. & 3 & 2 & 8,00 & 0,63 & 3,00 & 0,32 & 0,02 & 0,18 & 1,14 & 0,50 \\
\hline Maytenus floribunda & 3 & 2 & 8,00 & 0,63 & 3,00 & 0,32 & 0,01 & 0,12 & $\begin{array}{l}1,08 \\
1,0\end{array}$ & 0,45 \\
\hline Aspidosperma pyrifolium & 3 & 2 & 8,00 & 0,63 & 3,00 & 0,32 & 0,01 & 0,08 & 1,04 & 0,40 \\
\hline Commiphora sp. & 2 & 2 & 8,00 & 0,63 & 2,00 & 0,22 & 0,02 & 0,19 & 1,04 & 0,40 \\
\hline Cavanillesia arborea & 1 & 1 & 4,00 & 0,32 & 1,00 & 0,11 & 0,05 & 0,52 & 0,94 & 0,63 \\
\hline Ximenia americana & 2 & 2 & 8,00 & 0,63 & 2,00 & 0,22 & 0,01 & 0,07 & 0,92 & 0,29 \\
\hline Coutarea sp. & 2 & 1 & 4,00 & 0,32 & 2,00 & 0,22 & 0,01 & 0,09 & 0,62 & 0,30 \\
\hline Callisthene fasciculata & 1 & 1 & 4,00 & 0,32 & 1,00 & 0,11 & 0,01 & 0,13 & 0,55 & 0,23 \\
\hline Machaerium angustifolium & 1 & 1 & 4,00 & 0,32 & 1,00 & 0,11 & 0,01 & 0,13 & 0,55 & 0,23 \\
\hline Rhamnidium elaeocarpum & 1 & 1 & 4,00 & 0,32 & 1,00 & 0,11 & 0,01 & 0,10 & 0,52 & 0,21 \\
\hline Enterolobium contortisiliquum & 1 & 1 & 4,00 & 0,32 & 1,00 & 0,11 & 0,01 & 0,09 & 0,51 & 0,20 \\
\hline Terminalia argentea & 1 & 1 & 4,00 & 0,32 & 1,00 & 0,11 & 0,01 & 0,09 & 0,51 & 0,20 \\
\hline Maclura tinctoria & 1 & 1 & 4,00 & 0,32 & 1,00 & 0,11 & 0,01 & 0,08 & $\begin{array}{l}0,50 \\
.0 .6\end{array}$ & 0,19 \\
\hline Bauhinia acuruana & 1 & 1 & 4,00 & 0,32 & 1,00 & 0,11 & 0,00 & 0,03 & 0,46 & 0,14 \\
\hline Machaerium stipitatum & 1 & 1 & 4,00 & 0,32 & 1,00 & 0,11 & 0,00 & 0,03 & 0,45 & 0,13 \\
\hline Platypodium elegans & 1 & 1 & 4,00 & 0,32 & 1,00 & 0,11 & 0,00 & 0,02 & 0,45 & 0,13 \\
\hline Acacia glomerosa & 1 & 1 & 4,00 & 0,32 & 1,00 & 0,11 & 0,00 & 0,02 & 0,45 & 0,13 \\
\hline Total & 924 & & $1.264,0$ & 100,00 & 924,00 & 100,00 & 9,92 & 100,00 & 300,00 & 200,00 \\
\hline
\end{tabular}


Quadro 3 - Índice de diversidade de Shannon-Wienner $\left(\mathrm{H}^{\prime}\right)$ e equabilidade $(\mathrm{J}$ ') encontrado em áreas de floresta estacional decídua

Table 3 - Index of diversity of Shannon-Wienner ( $\left.\mathrm{H}^{\prime}\right)$ and Evenness ( $\mathrm{J}$ ') found in seasonal deciduous forest areas

\begin{tabular}{|c|c|c|c|c|c|}
\hline Autor & $\begin{array}{l}\text { Riqueza de } \\
\text { Espécies (S) }\end{array}$ & $\begin{array}{l}\text { Índice de Shannon- } \\
\text { Wienner (H') }\end{array}$ & $\begin{array}{l}\text { Equabilidade } \\
\text { de Pielou } \\
\left(\mathrm{J}^{\prime}\right)\end{array}$ & $\begin{array}{c}\text { Densidade } \\
\left(\mathrm{n}^{\mathrm{o}} \text { indivíduos } / \mathrm{ha}\right)\end{array}$ & $\begin{array}{c}\text { Área Basal } \\
\left(\mathrm{m}^{2} / \mathrm{ha}\right)\end{array}$ \\
\hline Este trabalho & 48 & 2,99 & 0,77 & 924 & 9,9 \\
\hline Silva \& Scariot (2003) & 52 & 3,19 & 0,81 & 861 & 18,6 \\
\hline Silva et al. (2001) & 36 & 2,99 & 0,83 & 536 & 8,4 \\
\hline Scariot \& Sevilha (2000) & 44 & 2,98 & 0,79 & 591 & 23,2 \\
\hline Scariot \& Sevilha (2000) & 48 & 3,03 & 0,78 & 674 & 24,5 \\
\hline Scariot \& Sevilha (2000) & 48 & 2,99 & 0,77 & 688 & 28,3 \\
\hline Ivanauskas \& Rodrigues (2000) & 54 & 3,00 & 0,70 & 894 & 29,7 \\
\hline
\end{tabular}

A densidade foi maior que as encontradas em outras áreas de floresta estacional decídua sobre solo e sobre afloramentos de calcário, porém os indivíduos são de menor porte, com área basal menor que as encontradas na mesma região estudada.

As alturas dos indivíduos amostrados foram distribuídas em classes com intervalo de $2 \mathrm{~m}$. A distribuição seguiu o padrão sigmóide das florestas tropicais. A maioria dos indivíduos $(93,8 \%)$ apresentou altura entre de 2 e $10 \mathrm{~m}$. Os indivíduos acima de $12 \mathrm{~m}$ de altura somaram 6,2\% do total, tendo a árvore mais alta atingido $22 \mathrm{~m}$.

É importante a preservação das áreas de florestas estacionais decíduas sobre afloramentos de calcários na região, dado ao pouco conhecimento desse tipo de vegetação e por elas estarem localizadas em áreas onde práticas de manejo, como o uso do fogo, ainda são utilizadas para formação de pastagens e expansão da fronteira agrícola, tornando-se uma ameaça a essas áreas. Em algumas regiões, a exploração de calcário tem provocado a erradicação desse tipo de vegetação.

Os afloramentos são muito importantes para a preservação da biodiversidade remanescente, uma vez que apresentam espécies de valor econômico e endêmicas, devendo ser ressaltado que a dificuldade de acesso e a ocorrência de algumas poucas florestas sobre áreas planas têm limitado a sua exploração.

\section{AGRADECIMENTOS}

Ao proprietário da fazenda Canadá, Sr. Antônio Ângelo, pela permissão para trabalharmos em suas terras. Ao Benedito Alísio da S. Pereira (IBGE), Bruno Machado
T. Walter e Alexandre Bonesso Sampaio (Cenargen) e à professora Carolyn Barnes Proença (UnB), pela identificação de algumas espécies. Ao Nilton F. Barbosa e Cláudio Vieira de Melo, pelo apoio na amostragem. Ao PRONABIO/PROBIO/BIRD/GEF/MMA/CNPq e Embrapa/Cenargen, pelo financiamento do projeto.

\section{REFERÊNCIAS BIBLIOGRÁFICAS}

BIERREGAARD Jr., R. O. et al. The biological dynamics of tropical rainforest fragments. Bioscience, v. 42, p. 859866, 1992.

GERHARDT, K. Seedling development of four tree species in secondary tropical dry forest in Guanacaste, Costa Rica. 1994. $43 \mathrm{f}$. Comprehensive Summaries of Uppsala Dissertations. Faculty of Science and Technology, 1994.

\section{FUNDAÇÃO INSTITUTO BRASILEIRO DE} GEOGRAFIA E ESTATÍSTICA- IBGE. Zoneamento ambiental e agroecológico do Estado de Goiás: região nordeste. Rio de Janeiro: 1995. 178 p. (Estudos e pesquisas em geociências, 3 ).

IVANUSKAS, N. M.; RODRIGUES, R. R. Florística e fitossociologia de floresta estacional decidual em Piracicaba, São Paulo, Brasil. Revista Brasileira de Botânica, v. 23, n. 3, p. 291-304, 2000.

LOVEJOY, T. E. et al. Ecological dynamics of tropical forest fragments. In: SUTTON, S. L.; WHITMORE, T. C.; CHADWICK, A. C. (Eds.) Tropical Rain Forest: Ecology and Management. Oxford: Blackwell Scientific Publication, 1983. p. $377-384$.

MAGURRAN, E. A. Ecological diversity and its measurement. Princeton: Princeton University Press, 1987. $192 \mathrm{p}$. 
MECENAS, V. V.; LEITE, F. Q.; CARDOSO, E. S. Composição florística de florestas sobre solo calcário, na área de proteção ambiental (APA) de Cafuringa, Brasília-DF. In: Forest'96 - 4., SIMPÓSIO INTERNACIONAL SOBRE ECOSSISTEMAS FLORESTAIS. Brasília: 1996. p. 326-327.

MULLER-DOMBOISD.; ELLENBERG, H. Aims and methods of vegetation ecology. New York: Wiley, 1974. $546 \mathrm{p}$.

PEDERSOLI, J. L.; MARTINS, J. L. A vegetação dos afloramentos de calcáreo. Oreades, v. 5, p. 27-33, 1972.

PEDRALLI, G. Florestas secas sobre afloramentos de calcário em Minas Gerais: florística e fisionomia. Bios, v. 5, n. 5 , p. 81-88, 1997.

SCARIOT, A.; SEVILHA, A. C. Diversidade, estrutura e manejo de florestas deciduais e as estratégias para a conservação. In: CONGRESSO NACIONAL DE BOTÂNICA, 51., 2000, Brasília. Tópicos Atuais em Botânica. Brasília: 2000. p. 183-188.

SILVA, L. Á.; SCARIOT, A. Composição florística e strutura da comunidade arbórea em uma floresta estacional decídua em afloramento calcário (Fazenda São José, São Domingos-GO, Bacia do Rio Paranã). Acta Botânica Brasílica, v. 17, n. 2, p. 307-315, 2003.

SILVA, L. Á.; SCARIOT, A. Levantamento da comunidade arbórea de floresta estacional decidual sobre afloramento calcário (Fazenda São Vicente, São Domingos - GO). In: CONGRESSO DE ECOLOGIA DO BRASIL: AMBIENTE E SOCIEDADE, 5., 2001, Porto Alegre. Resumos... Porto Alegre: Universidade Federal do Rrio Grande do Sul, 2001. p. 190.
SILVA, L. Á.; SCARIOT, A.; SEVILHA, A. C. Comunidade arbórea da floresta estacional decidual em afloramento calcário: São Domingos-GO. In: CONGRESO LATINO AMERICANO DE ECOLOGÍA, 5., 2001, San Salvador de Jujuy, Argentina. Anais... San Salvador de Jujuv: 2001. não-paginado.

SKOLE, D.; TUCKER, C. Tropical deforestation and habitat fragmentation in the Amazon: satellite data from 1978 to 1988. Science, v. 260, p. 1905-1910, 1993.

SPIEGEL, M. R. Estatística. São Paulo: McGraw-Hill, 1976. 357 p.

VELOSO, H. P.; RANGEL FILHO, A. L. R.; LIMA, J. C. A. Classificação da vegetação brasileira adaptada a um sistema universal. Rio de Janeiro: IBGE, Departamento de Recursos Naturais e Estudos Ambientais, 1991. 123 p.

WERNECK, M. S.; FRANCESCHINELLI, E. V.;

TAMEIRÃO-NETO, E. Mudanças na florística e estrutura de uma floresta decídua durante um período de quatro anos (1994-1998), na região do Triângulo Mineiro, MG. Revista Brasileira de Botânica, v. 23, n. 4, p. 401-413, 2000.

WHITMORE, T. C. Tropical forest disturbance, disappearance, and species loss. In: LAURANCE, W. F.; BIERREGAARD, R. O. (Eds.) Tropical forest remnants, ecology, management, and conservation of fragmented communities. Chicago: University of Chicago Press, 1997. p. 3-12. 